\title{
La Filosofía de la Ocupación Humana y el Paradigma Social de la Ocupación. Algunas reflexiones y propuestas sobre epistemologías actuales en Terapia Ocupacional y Ciencias de la Ocupación.
}

\section{The Philosophy of Human Occupation and the Social Paradigm of the Occupation. Some reflections and suggestions on current epistemologies in Occupational Therapy and Occupational Science.}

\author{
Rodolfo Morrison J. ${ }^{1}$, Daniela Olivares A. ${ }^{2}$, Diego Vidal M. ${ }^{3}$
}

\section{Resumen}

Este ensayo corresponde al establecimiento de un posicionamiento epistemológico dentro del paradigma actual de la Terapia Ocupacional, el que hemos denominado Paradigma Social de la Ocupación. Dirigimos esta mirada desde la práctica profesional y desde el análisis crítico del conocimiento y posicionamiento científico de la Terapia Ocupacional y las Ciencias de la Ocupación. Dicho análisis se encuentra bajo una perspectiva históricaepistemológica (desde las raíces de la disciplina) y contextual (coherente con la cultura y la región), apoyada en la Filosofía de la Ciencia.

La propuesta se desarrolla a partir de la revisión de los diversos y sucesivos posicionamientos epistémicos de la Filosofía de la Ciencia, desde los orígenes de la disciplina hasta nuestros días, realizando un meta-análisis, sobre la influencia de éstos en la conformación y desarrollo de la profesión. Para lo anterior, nos situamos en una rama de la filosofía de la ciencia, la que nos permite una reflexión de segundo orden sobre nuestra disciplina que hemos llamado Filosofía de la Ocupación Humana.

Como primer ejercicio meta analítico, realizamos un análisis de segundo orden desde la Filosofía de la Ocupación Humana, en relación con la categorización hecha por Kielhofner sobre la práctica de la terapia ocupacional, según la cual se describen tres paradigmas que caracterizan a la Terapia Ocupacional en periodos históricos diferentes, influenciando la práctica clínica, los valores, los objetivos y la evidencia científica que han sustentado el quehacer profesional.

Siguiendo esta línea, aparece un cuarto paradigma -Paradigma Social de la Ocupación-, el que se configura luego que la perspectiva única de la ocupación como fenómeno individual (tercer paradigma) no fuese concordante con las necesidades de las sociedades ni comunidades, ni respondiese de manera completa a los nuevos campos de acción de los terapeutas ocupacionales. Según esto, concluimos la necesidad de adoptar para la disciplina, una epistemología y un pensamiento complejos, según lo definido por Edgar Morin, para poder continuar con el desarrollo teórico de la profesión que permita dar respuestas a los nuevos desafíos surgidos en nuestros sujetos de atención y sus contextos.

Palabras clave: epistemología, terapia ocupacional social, filosofía de la ocupación humana, paradigma, paradigma social de la ocupación, reflexión de segundo orden, ciencias de la ocupación.

1 Máster en Lógica y Filosofía de la Ciencia, Univ. de Salamanca, Terapeuta Ocupacional y Lic. en Ciencias de la Ocupación, Univ. Austral de Chile. Coordinador Equipo de Tratamiento Ambulatorio-Comunitario Plan de Intervención Integral La Legua. SENDA. Contacto: morrison.rodolfo@gmail.com.

2 Lic. Cs. de la Ocupación y Terapeuta Ocupacional por la Univ. de Chile, Especialista en Integración Sensorial, University of Southern California y Western Psychological Services. Académica Escuela de Terapia Ocupacional, Universidad Austral de Chile. Contacto: danielaolivares@uach.cl.

3 Lic. en Ciencias de la Ocupación y Terapeuta Ocupacional por la Univ. Austral de Chile. Diplomado en Salud Mental y Psiquiatría Comunitaria por la Univ. de Chile. Terapeuta Ocupacional Hospital de Día Valdivia y Unidad de Hospitalización de Corta Estadía en Medio Privativo de Libertad CIP-CRC Valdivia. Contacto: dvidalmadrid@gmail.com. 


\begin{abstract}
This essay corresponds to the establishment of an epistemological position inside the current paradigm of the occupational therapy, which we call Social Paradigm of Occupation. We are making this proposal from professional practice and critical analysis of the scientific knowledge and position of the occupational therapy and the occupational science. This analysis is located in a historical, epistemological (from the roots of the discipline) and contextual perspective (consistent with the culture and the region) supported by the Philosophy of Science.

The proposal is developed after reviewing the various successive epistemological positions of the Philosophy of Science, from the beginnings of the discipline until our time, doing a meta-analysis about the influence of these positions in the formation and development of the profession. To that end, we position ourselves in a particular branch of the Philosophy of Science that allow us to do a second order reflection from the Human Occupation Philosophy, regarding the categorization elaborated by Kielhofner about the practice of occupational therapy. This proposal describes three paradigms that characterize the Occupational Therapy in three different historical periods, which have been influencing clinical practice, values, objectives and the scientific evidence that have supported the professional work.
\end{abstract}

Following this line, we conclude that it is necessary to the discipline to adopt a complex epistemology and thinking, according to the definition proposed by Edgar Morin. This way it would be possible to continue the theoretic development of the profession that allows us to answer to the new challenges that rise from the subjects we work with and their contexts.

Key words: epistemology, social occupational therapy, philosophy of human occupation, paradigm, social paradigm of occupation, reflection of second-order, occupational science.

\author{
"El desafio de la complejidad \\ es el de pensar complejamente \\ como metodología de acción cotidiana, \\ cualesquiera sea el campo en el que \\ desempeñemos nuestro quehacer."
}

Marcelo Pakman ${ }^{4}$

\title{
Introducción
}

Este trabajo se encuadra como la síntesis de reflexiones realizadas por los autores y la autora durante nuestro período de formación en la Universidad Austral de Chile ${ }^{5}$ (como terapeutas ocupacionales Rodolfo y Diego, y como docente Daniela). Tras variadas discusiones con otros terapeutas ocupacionales, nos surge la necesidad de sistematizar nuestras conclusiones para

4 Marcelo Pakman (31, p. 14).

5 Algunas de esas reflexiones se materializaron en los siguientes trabajos inéditos: - Vidal y Morrison. Posicionamiento epistemológico en el paradigma emergente: un sustento para la terapia ocupacional. Ponencia presentada en 2006 (nov) en la IV Jor de Est de TO. Univ Mayor. Stgo, Ch.; · Olivares, Vidal y Morrison. De la Filosofía a la Ciencia de la Ocupación: Propuesta Epistemológica Para la Práctica Contemporánea. Póster presentado en 2007 (sep) en el 7mo Congr Latinoam y 7mo Congr Arg de TO. Mar del Plata, Arg; · Vidal y Morrison. Ontología, Epistemología y Lógica: Filosofía de la Ocupación Humana. Ponencia presentada en 2007 (ago) en el II Congr Nac de Est Univ de TO. Val, Ch, resumen disponible en: http://www.boletinto.cl/pdf/016.pdf; · Vidal y Morrison. Análisis ontológico de la ocupación humana. [Sem de Título dirigido por Daniela Olivares]. Val: Esc de TO, Univ Austral de Ch, 2009; · Vidal y Morrison. Ontología de la Ocupación Humana. Pasos hacia un nuevo Paradigma. Ponencia presentada en 2010 (may) en el XV Congr Mund de la Feder Mund de TO. Stgo, Ch, 2010, resumen disponible en: http://www.wfot.org/wfot2010/program/pdf/1469.pdf. 
fomentar la discusión epistemológica y las reflexiones sobre nuestra disciplina, tanto a nivel académico, como en las intervenciones profesionales.

Por lo anterior, el objetivo del siguiente ensayo es aportar a las reflexiones críticas sobre la práctica profesional y sobre la conformación del conocimiento. Para ello, estructuramos el escrito de la siguiente manera: primero explicitamos el posicionamiento teórico de los autores y la autora respecto al lugar desde el que realizamos las reflexiones, el que hemos llamado Filosofía de la ocupación humana; luego reflexionamos en torno a los paradigmas presentes en la disciplina, identificando las principales epistemologías para comprender a la misma, para después realizar una comparación entre dos maneras de concebir el quehacer de la Terapia Ocupacional y las Ciencias de la Ocupación en la actualidad (incluyendo el ejercicio profesional y la construcción de conocimiento). Finalmente, sugerimos algunos posicionamientos teóricos que se enmarcan en el actual paradigma de la profesión, el que hemos denominado Paradigma Social de la Ocupación.

\section{Filosofía de la ciencia}

El análisis reflexivo sobre la ciencia y su práctica, como también sobre las disciplinas profesionales que sustenta y reproduce, han sido objeto de atención desde muchas décadas para científicos(as) y filósofos(as). Esto ha derivado en la constitución de un campo disciplinar autónomo y delimitado, denominado Filosofía de la ciencia, el que reflexiona desde un meta-análisis sobre la conformación, estructura y dinámica del conocimiento científico, y sobre los fines y condiciones de la práctica científica (1); (2); (3); (4).

Existe acuerdo entre los principales autores en filosofía de la ciencia, que sus inicios como disciplina consolidada se remontan a principios del siglo XX con la configuración del Círculo de Viena en 1929 (1); (2). Este grupo científico-filosófico buscó elaborar explicaciones sobre la estructura del conocimiento científico, desde una posición derivada del Positivismo lógico conocida como Neopositivismo (ibíd.). Desde este enfoque, la ciencia comienza a ser estudiada por medio de la elaboración de meta-teorías que, primero, dan cuenta del conocimiento en sí mismo, y posteriormente, de la práctica científica ${ }^{6}$ (2).

En un comienzo, dentro del Círculo de Viena se considera al conocimiento como un proceso acumulativo e independiente del(a) observador(a), en donde se identifican Leyes empíricas y Leyes teóricas, las que explicarían cómo las y los científicos estructuran este conocimiento. Las leyes empíricas serían las relacionadas con los sentidos y se expresarían en "términos observables", es decir, bajo la creencia que los objetos existen independientemente del observador, como la temperatura, la presión, etc. Por otro lado, las leyes teóricas explicarían cómo funcionan los fenómenos de la naturaleza desde términos no observables, como átomos, moléculas, etc. (5).

Con el paso del tiempo se pone en tela de juicio la manera de concebir a la ciencia en

6 Es importante señalar que esta dicotomía entre "contenido" de la ciencia versus "práctica" científica, es una discusión que se mantiene hasta nuestros días, y que ha transitado con diferentes nomenclaturas, quizás la más característica es la del contexto de justificación y el contexto de descubrimiento propuesta por Hans Reichenbach en 1938 (9). 
dicotomías observables/teóricas, principalmente porque las "leyes empíricas" también responden a conceptualizaciones teóricas previas que se emplean para dar cuenta de lo que entendemos por realidad.

Un quiebre importante en la filosofía de la ciencia ocurre con la publicación del libro $\mathrm{La}$ estructura de las revoluciones científicas de Thomas Kuhn en 1962. Por un lado, en este libro se re-definen las maneras establecidas para comprender el conocimiento científico, otorgándole valor al "hacer" de los y las científicas, junto con las circunstancias sociales en las que se encuentran, debido a que formas más "competentes" de conocimiento científico se superponen al conocimiento anterior (6). Por otro lado, la ciencia transita desde una concepción lineal a una cíclica, el conocimiento no sería acumulativo, sino que se "reformaría" en cada revolución científica. Luego de sucesivas "revoluciones", la manera de "hacer", y por ende, de "comprender" la ciencia, cambia según la configuración de Paradigmas. Estos paradigmas, que pueden entenderse como criterios de validación de la verdad, permutan y se transforman en función de múltiples factores, lo que afecta a la práctica científica (6).

La filosofía de la ciencia inicial buscaba la comprensión del conocimiento científico aislado e independiente de las características contextuales en las que se produce, es decir, para los neopositivistas del Círculo de Viena, el conocimiento es válido por su compleja estructura y composición lógica, en la que los factores contextuales no son, en primera instancia, determinantes (2). A partir de los cuestionamientos de Kuhn, se identifica que estos factores sí son determinantes en la práctica científica, por lo que resulta crucial comprenderlos y relacionarlos con la elaboración de meta-teorías que den cuenta de ello. De este modo, la ciencia ya no sólo se entiende como el conocimiento aislado, sino también como una práctica social y humana, por lo que es imposible aislar los valores, la política y toda ideología del conocimiento científico (3); (4); (7); (8).

Los debates actuales en filosofía de la ciencia comienzan a desvanecer la distinción dicotómica de contexto de justificación (estructura teórica del conocimiento) y contexto de descubrimiento (características "externas" a la ciencia que podrían influir en su desempeño, como la política, los valores, etc.), conceptos empleados para dar cuenta que el conocimiento permanece aislado y no se relaciona con características que, arbitrariamente, se distinguen y se separan de la ciencia. Una de las posiciones más actuales propone que la ciencia es, como hemos señalado, una práctica social y humana y produce epistemologías o formas de conocer construidas -acordadas y consensuadas por métodos igualmente acordados- que también dependen del contexto para ser formuladas (4); (7).

Además de la recién descrita perspectiva histórica-epistemológica sobre la filosofía de la ciencia, podemos realizar otra distinción sobre ella relacionada con sus objetos de análisis y por ende, dividirlas en categorías. Esta puede ser comprendida desde una manera general, es decir, identificando leyes que respondan a la configuración del conocimiento científico de una manera no específica, y desde una manera particular, en la que disciplinas determinadas y parcializadas, que producen conocimiento científico, son estudiadas. De esta forma, disciplinas como la matemática, física, psicología, entre otras, poseen reflexiones de segundo orden, es decir, de un nivel de abstracción mayor en relación a si mismas ${ }^{7}$.

7 Es importante señalar que la filosofía de la ciencia neopositivista, o entendida también como La concepción heredada, comienza a configurarse como tal desde la Filosofía de la física, y de la Filosofía de las 
En ambos contextos descritos, histórico-epistemológico y categórico, proponemos que la Terapia Ocupacional y las Ciencias de la Ocupación podrían ser estudiadas y analizadas desde una reflexión de segundo orden, en lo que hemos denominado Filosofía de la Ocupación Humana. De este modo, pretendemos facilitar la observación e interpretación de la ocupación como un fenómeno filosófico, a modo de proporcionar mayor profundidad en el análisis del ejercicio profesional y académico de ambas disciplinas.

\section{Filosofía de la ocupación humana}

Siguiendo este razonamiento, hemos señalado que la filosofía (general y específica) de la ciencia, reflexiona en torno a la estructura y dinámica del conocimiento científico y respecto a la práctica de los y las científicas, como también sobre las disciplinas aledañas y profesionalizadas que emplean al conocimiento científico como sustento de su quehacer.

En este último punto, el conocimiento científico producido por las Ciencias de la Ocupación (CO) y la Terapia Ocupacional (TO), también son un punto interesante de análisis y discusión para la filosofía específica de la ciencia, configurándose una Filosofía de la Ocupación Humana (10); (11); (12).

De este modo, proponemos que la Filosofía de la ocupación humana (FOH) no sólo formula reflexiones críticas al ejercicio disciplinar y sobre la producción de conocimiento científico de la TO y CO, sino también, desde su enfoque, podemos analizar críticamente el conocimiento que se pone en práctica, pensando sobre qué epistemologías podrían constituir un cuerpo de conocimiento acorde y contextualizado, que fortalezca el desempeño de los y las terapeutas ocupacionales de acuerdo al contexto actual de la disciplina.

Así, por medio de la $\mathrm{FOH}$, reflexionamos respecto a qué posicionamiento epistemológico podría corresponder al "actual" paradigma de la profesión, que pueda guiar la formación de nuevos profesionales en la disciplina y al cuestionamiento sobre las teorías empleadas en la práctica actual. Es necesario resaltar que esta reflexión no corresponde a un primer orden ${ }^{8}$ como lo es la TO y las $\mathrm{CO}$, es decir, sobre la puesta en práctica del conocimiento, como analizar la estructura de rutina de determinados usuarios, o las condiciones de injusticia ocupacional en determinadas comunidades, o los déficit sensoriales de niñas de un grupo específico (11); la FOH, en cambio, reflexiona en torno a "cómo" esos conocimientos de base son construidos y puestos en práctica por los y las terapeutas ocupacionales.

Desde la FOH observamos cómo los diferentes paradigmas de la profesión responden a

matemáticas (2).

8 Desde la categorización de los tipos lógicos de Bateson (43) una reflexión de primer orden corresponde al análisis o reflexión sobre el hacer o actuar de una disciplina desde sus ejes constituyentes, fundamentos teóricos o evidencia científica, mientras que un análisis de segundo orden, o también denominado metaanálisis, corresponde a identificar por qué y cómo esos fundamentos se relacionan, sirven o vinculan con aquella disciplina. Desde esta perspectiva, el análisis de primer orden representaría el "qué observamos", mientras que el de segundo orden al "cómo observamos". Por ello, podemos concluir que para reflexionar sobre cómo miramos la realidad, es necesario un nivel de abstracción mayor que englobe a la categoría anterior o inicial. 
epistemologías y posteriormente a prácticas (o viceversa) resaltando ejemplares (muestras o ejemplos del (o los) eje(s) centrales del paradigma) y procesos históricos (eventos que dan cuenta de los procesos o revoluciones) (6). Por lo anterior, y como describiremos más adelante, en el Paradigma social de la ocupación es necesario desarrollar un posicionamiento teórico coherente con el "tipo" o "estilo" de práctica profesional que se ejecuta.

Sin embargo, para comprender este argumento, consideramos necesario revisar los paradigmas por los que ha atravesado el ejercicio profesional y realizar un paralelo entre una epistemología reduccionista y una basada en la complejidad.

\section{Paradigmas en la disciplina}

Diversos(as) autores(as) en TO y $\mathrm{CO}$ han reflexionado respecto a la práctica profesional. Kielhofner (13) por ejemplo, analiza la TO desde una visión histórica definiendo y describiendo tres paradigmas que han influenciando la práctica clínica, los valores, los objetivos y la evidencia científica que sustenta su quehacer profesional. Lo anterior, desde un análisis de primer orden que dispone los "eventos" en categorías que son definidas por la historicidad y la historiografía, y no desde su meta-contenido, carece de una reflexión crítica respecto a la construcción de ese conocimiento?.

Ideas conducentes a la constitución de la terapia ocupacional y el Pre-paradigma de la Ocupación

Previo a la conformación del primer paradigma, diversas teorías e ideologías comienzan a dar forma a la disciplina. Entre las ideas conducentes a la conformación de la disciplina, se distinguen tres principales, las que dan forma a la naciente TO. De esta manera y observando desde una perspectiva de segundo orden, este primer paradigma se configura como el inicio epistemológico de la TO (fines del siglo XIX), el que se basó en fundamentos teóricos como la Filosofía Pragmatista (15), el Tratamiento Moral (16) y el Moviendo de Artes y Oficios (17); (18), epistemologías que fundamentaban la intervención terapéutica, o el "tratamiento ocupacional", en concepciones filosóficas opuestas a las "visiones de mundo" imperantes en aquella época, como el fundamentalismo teórico, la discriminación de clases y desigualdad social, y la industrialización (15); (18); (19), características no planteadas por Kielhofner en el denominado Pre-paradigma de la Ocupación (13).

El contexto socio-histórico en el que comienza a gestarse este paradigma, se vincula con la Segunda revolución industrial (18), comprendida entre los años 1850 y 1914 (decreciendo como foco con la Primera Guerra Mundial) y la Segunda ola del feminismo, momento en que el poder de las mujeres se expande por los Estados Unidos liberándose del yugo masculino (20).

9 Un ejemplo de esto es que en el análisis de Kielhofner (13), la variable de género, si bien es mencionada, no se realiza un juicio crítico respecto a cómo la epistemología de la profesión también se ha visto teñida desde esta perspectiva (14). 
En este pre-paradigma, la ocupación se gestaba como el eje central de la disciplina, comenzando a constituirse como tal a comienzos del siglo XX, consolidándose en 1917 con la fundación de la National Society for the Promotion of Occupational Therapy (18).

Especialmente en este período, fueron las mujeres, influenciadas por las ideologías antes mencionadas, las encargadas de expandir la profesión (14); (17), destacándose la primera técnica de TO denominada Entrenamiento en hábitos, elaborada por Eleanor Clarke Slagle, una de las fundadoras de la disciplina. Esta técnica serviría como fundamento de la novel profesión frente al mundo biomédico, especialmente en psiquiatría (21). Slagle también otorgó un enfoque centrado en la integración de personas "diferentes" (con o sin discapacidad) en la sociedad (22). Por otro lado, la primera terapeuta ocupacional Susan Tracy (17), comenzó con la formación de profesionales, bajo las influencias de los pragmatistas John Dewey y William James, practicando "tratamiento ocupacional" a "pacientes" que lo necesitaran en hospitales (26).

Otro de los eventos importantes de este período estuvo a cargo de Adolf Meyer, quien escribió $^{10}$ las bases filosóficas sobre la TO, considerando una visión integral del ser humano y aplicando los principios éticos del tratamiento moral (16) y la filosofía pragmatista (15), resaltando el rol terapéutico de la ocupación (24), idea heredada desde el movimiento de artes y oficios (18).

Como parte de un gran grupo de personas que consideraban a la ocupación como un agente de promoción, tratamiento y restauración de la salud, Slagle, Tracy y Meyer, comienzan a dar marco y contexto a esta novedosa terapia, la que tendría un auge significativo durante la Primera y Segunda Guerra Mundial. Así, los enfoques iniciales de TO prosperaban considerablemente.

\section{Paradigma Mecanicista}

Debido a los tránsitos paradigmáticos generados por el contexto social, ideológico, cultural, político y económico, con todo lo que ello implica, la manera de comprender a la TO cambia. El auge del Neopositivismo lógico (1) limita el concepto de ciencia a una reducible en variables y dicotomías, por lo que los fundamentos holistas y humanistas (en origen pragmatistas y adoptados por los y las primeras terapeutas ocupacionales) no tienen cabida, ni tampoco, una explicación coherente con la manera de concebir la ciencia, ni las disciplinas que no respaldan su desempeño en métodos cuantitativos y reduccionistas.

Lo anterior impulsa a las sucesivas generaciones de terapeutas ocupacionales, a la búsqueda de validación profesional en las ciencias biomédicas. Es así como, bajo un Paradigma

10 Es importante resaltar que Meyer fue quien escribió parte de las bases filosóficas de la TO, debido a que estas ya existían y se habían configurado recientemente. Sin embargo, no fueron explicitadas en su totalidad, lo que implicó que parte de ellas no fuesen consideradas durante muchos períodos en la historia de la disciplina. Desde esta óptica, consideramos que es un error considerar a Meyer como "la" figura que "instauró" las bases de la disciplina, y que más bien, gracias al paradigma androcéntrico, fue uno de los varones más reconocidos (14); (22); (23). 
Mecanicista, la TO es considerada como una disciplina ligada (y jerarquizada) a la Medicina, fundamentado su intervención en técnicas paliativas para reducir incapacidad y centrada en la funcionalidad de sistemas biológicos e intrapsíquicos (26).

Durante la Segunda Guerra Mundial, los y las terapeutas ocupacionales que trabajaron en la rehabilitación de soldados, estaban bajo las indicaciones del personal médico-militar, comenzando a posicionarse este nuevo enfoque de la profesión, centrado en la funcionalidad y la consecución de objetivos concretos en el menor tiempo posible, optimizando recursos, desde una perspectiva mecanicista. La ocupación deja de ser el eje de la intervención en la profesión, constituyéndose un nuevo eje central: la función (ibíd.).

Los preceptos de Slagle, Tracy y Meyer, entre otros, toman diferente curso; la TO desarrolla un nuevo enfoque, potenciando una visión científica neopositivista apoyada en las ciencias biomédicas. Luego de la Segunda Guerra Mundial, la TO necesita seguir su curso, por lo que este nuevo enfoque, insipiente en ese momento, se refuerza para constituirse como una disciplina de apoyo médico (ibíd.).

La TO desarrolló teorías e hipótesis sobre cómo la "ocupación" puede servir para lograr objetivos específicos dentro de otros campos del conocimiento. Margaret Rood entrega una visión desde una perspectiva musculoesquelética, mientras que los trabajos iniciales de Jean Ayres aportaban desde el enfoque neurobiológico. Ambas perspectivas, sumadas a las contribuciones de Gail Fidler, quien argumentó el rol de la TO en la resolución de los conflictos intrapsíquicos, constituyen una gran fuente del conocimiento científico (desde otros campos fuera de TO) que justificó su práctica de la TO por muchas décadas (13).

\section{Paradigma de la Ocupación}

Una siguiente revolución científica, esta vez entre la Modernidad y la Postmodernidad como fenómeno mundial (27), lleva a un cambio desde un paradigma neopositivista a uno Interpretacionista, y tras los conflictos de identidad de los TO -como lo conocido por práctica subterránea (28)- le exige al desarrollo del conocimiento y la práctica de la TO la búsqueda de nuevas concepciones y enfoques pertinentes, para así responder a las necesidades de salud y calidad de vida de las personas.

La aparición de Mary Reilly favorece un giro en el quehacer de la disciplina. Reilly hace énfasis en el volver a los fundamentos iniciales de la profesión, centrándose en la ocupación como su eje principal. El comportamiento ocupacional, teoría elaborada por Reilly, invita a terapeutas ocupacionales a reflexionar sobre su desempeño y la manera de comprender a sus usuarios (13). Ayres continúa con el desarrollo de su teoría, otorgándole un nuevo enfoque centrado en la ocupación. Los aportes de Jean Ayres y Mary Reilly son considerados como las primeras teorías centradas en la ocupación dentro del nuevo paradigma, las que son utilizadas por terapeutas ocupacionales, que retoman los fundamentos iniciales de la profesión (ibíd.).

Por su parte, David Nelson establece reflexiones sobre la concepción de ocupación, desde la University of Southern California, realizando la diferencia entre Forma y Desempeño ocupacional; este enfoque será clave en la posterior comprensión de la ocupación humana. 
Paralelamente se inicia el Doctorado en Ciencias de la Ocupación, influenciado por las investigaciones de Reilly y las consideraciones de Yerxa (13); (26).

Kielhofner y Burke, propulsores del Modelo de Ocupación Humana, se basan en las premisas iniciales de Reilly, considerando conceptualizaciones teóricas de Nelson y establecen que la TO debe tener un fundamento como ciencia aplicada (13). Paralelamente, el Modelo Canadiense de Desempeño Ocupacional, elaborado por la Asociación Canadiense de Terapia Ocupacional, considera la ocupación desde múltiples perspectivas, incluyendo la Espiritualidad como factor nuclear (42). Estos dos modelos principales son utilizados en la actualidad, fortaleciendo el desempeño de terapeutas ocupacionales en diferentes partes del mundo, siendo ejemplares de modelos centrados en la Ocupación (12); (13); (26).

Por lo antes señalado, dentro de la práctica de la TO se describe el surgimiento de un nuevo paradigma científico, centrado en la Ocupación Humana como eje central, la que valida su quehacer en este nuevo contexto histórico, configurándose el Paradigma de la Ocupación, así también, comienzan a gestarse y fortalecerse las Ciencias de la Ocupación (ibíd.).

Desde un paradigma más amplio, Postmoderno y también Postindustrial (29), la Ocupación Humana, en este tercer paradigma, se concibe como un fenómeno individual y subjetivo, destacándose el desarrollo de modelos de intervención como el conocido Modelo de Ocupación Humana, continuando el desarrollo de las Ciencias de la Ocupación (30), comprendiéndose además, a la ocupación humana como un fenómeno cada vez más complejo, como las propuestas teóricas de Ann Wilcock sobre la Naturaleza Ocupacional del Ser Humano (29).

En la actualidad, la ocupación humana ya no es concebida solamente como un fenómeno individual, comenzando a romper la creencia que "impone" modelos teóricos desde países desarrollados a realidades locales en vías de desarrollo. Se potencia la adaptación y elaboración local y se concibe a la Ocupación como fenómeno social, lo que responde a un nuevo paradigma, el que hemos denominado Paradigma Social de la Ocupación, que considera a la ocupación como un fenómeno sistémico, complejo, económico, político, sanitario, cultural, social y coherente con la justicia y el bienestar de las comunidades (26) e inserto dentro de un nivel de abstracción mayor: el Paradigma de la Complejidad.

Como terapeutas ocupacionales consideramos que para posicionarse en este escenario, es necesario realizar un breve paralelo entre las epistemologías que se desprenden de estos paradigmas antes descritos, de modo de fortalecer el ejercicio profesional y la investigación científica desde una perspectiva actual y coherente con los nuevos desarrollos epistémicos. Por ello, a continuación reflexionamos al respecto.

\section{Epistemología Reduccionista versus Epistemología Compleja}

Como hemos planteado hasta el momento, desde la perspectiva de la FOH, el desarrollo teórico-metodológico de la TO ha estado influenciado no sólo por el desarrollo de la ciencia, 
sino por distintos contextos socio-político-históricos ${ }^{11}$.

Sumado a lo anterior, María Heloisa da Rocha Medeiros (32), plantea que, comparativamente con otras profesiones, la TO es una disciplina relativamente nueva, más aun en su desarrollo científico, por lo que no cuenta, hasta ahora, con una tradición de conocimientos propios y sistematizados que le permita tener un desarrollo epistemológico propio, generando en la actualidad cuestionamientos entre los y las terapeutas ocupacionales sobre qué tipo de ciencia "es" la TO, o cuáles son los límites de su área de conocimiento, iniciándose así una discusión epistemológica al respecto.

Medeiros define a la TO como “(...) área del conocimiento y práctica de salud, [que] se interesa por los problemas del (...) [ser humano] en su vida de actividades. En otras palabras, considera las actividades humanas como el producto y el medio de construcción del propio (...) [ser humano] y busca entender las relaciones que éste establece, mediante su actividad, en su condición de vida y salud." (ibíd. p.17).

Esta definición de la disciplina, así como otras que podemos encontrar en la literatura, contiene una serie de supuestos de base que están lejos de ser comprendidos fuera de conceptualizaciones teóricas, que como ya hemos dicho, son parte del contexto en el que son desarrolladas. Es por esto que cuando comenzamos a cuestionarnos sobre el posicionamiento epistemológico de la disciplina, desde perspectivas históricas y actuales, nos preguntamos por el alcance, los valores y los límites de las diferentes teorías en TO (32), lo que nos "remite también a cuestiones de cuño más filosófico que llevan a pensar qué es el (...) [ser humano], cuáles son los valores de su existencia, de su conocimiento, ya que toda discusión epistemológica se asienta en una decisión filosófica y antropológica." (ibíd. p. 19).

Continuando con la discusión, Medeiros señala que la TO se ha considerado "como una práctica bastante caótica, de estructura laxa" $(32$, p. 18), es decir "una práctica constituida por técnicas oriundas de diferentes concepciones y valoraciones de[1] (...) [ser humano] y sus actividades, de la salud y de la enfermedad, de la sociedad, formando una verdadera amalgama teórica-práctica" (ibíd. p. 18-19).

Considerando esta problemática, comenzamos a buscar respuestas apoyados en diferentes epistemologías, intentando comprender y explicar el actual estado de nuestra disciplina. Junto con la FOH, descubrimos a Edgar Morin, filósofo y sociólogo francés, considerado uno de los pensadores más importantes en la actualidad (41), quien nos desafía a posicionarnos en un pensamiento multidimensional, problematizador y complejo, propio del Paradigma de la Complejidad, en oposición al pensamiento vectorial, reduccionista y simplificador, coherente con el Paradigma Mecanicista, con base epistémica neopositivista propia de la Modernidad (31).

Esta actual transición paradigmática ${ }^{12}$ planteada por Morin (ibíd.), permite establecer cambios en el abordaje contemporáneo de las diversas necesidades actuales de la población, como la

\footnotetext{
${ }^{11}$ Ver figura 2.

12 La que correspondería a la configuración de un nuevo paradigma para comprender lo que entendemos por realidad. Nosotros comprendemos el Paradigma de la Complejidad diferente del Paradigma Postmoderno, debido a que éste último correspondería más bien a un período interparadigmático, desde la concepción kunhiana, más que a un paradigma en sí mismo (6).
} 
economía, la educación y la salud, entregando un análisis epistemológico que puede contribuir al desarrollo del conocimiento en general y de las $\mathrm{CO}$ y $\mathrm{TO}$ en particular.

Siguiendo las ideas de Morin, podemos establecer que bajo un Paradigma Mecanicista, se comprenden, abordan y resuelven los problemas en salud basados en la dicotomía saludenfermedad. Este enfoque conlleva a la construcción de sistemas de salud centrados en la asistencialidad y lo paliativo, o en palabras de Martínez, "a un encuadre negativo de los procesos salud enfermedad", es decir, relevando "en las personas, grupos y comunidades sus deficiencias, incapacidades, problemas, síntomas, dificultades, traumas, déficits, desórdenes, daños, vulnerabilidades, etc." (33, p. 16). En contraposición, podemos suponer que bajo el Paradigma de la Complejidad, los sistemas de salud podrían incluir, dentro de muchas variables, un "encuadre positivo"13, es decir, "hacer emerger en las personas, grupos y comunidades sus capacidades, competencias, habilidades, recursos y potencialidades" (ibíd.), fortaleciendo su calidad de vida.

La perspectiva mecanicista establece una fundamentación teórica del ejercicio profesional, describiendo lo que observamos por medio de variables solamente cuantificables y válidas en su demostración empírica, lo que se relaciona con la autoproclamada autoridad epistémica, la que se expresa con el término objetividad (11); (27).

El quehacer profesional desde una práctica construida en una epistemología reduccionista, se enfoca en la patología, la disfunción y la reparación, estableciendo además, una relación jerárquica y de poder hacia un usuario o usuaria pasiva (paciente), diferenciándose de una práctica construida bajo una epistemología compleja, enfocada en la persona, la ocupación y la inclusión social, que va hacia al encuentro de las necesidades individuales y colectivas en salud, implicando además, la horizontalidad y el fortalecimiento de las usuarias y usuarios en salud, en donde se incluye un enfoque de participación ocupacional social, entregando y aportando identidades y validación dentro de nuestra profesión (12); (26).

\section{Paradigma Social de la Ocupación}

La figura 1 representa los diferentes paradigmas en TO, incluyendo un estadio inicial que hemos denominado ideas conducentes a la conformación de la disciplina. En la figura 2, observamos los mismos paradigmas, pero insertos en un contexto ideológico más global, lo que permite comprender las diferentes teorías, que ya hemos mencionado, que se relacionan y dan forma a la TO, además de hacer hincapié en la perspectiva que empleamos para observar esto, la FOH. Ambas figuras pueden comprenderse como una síntesis teórica que convergen en el paradigma social de la ocupación. En ellas podemos observar los inicios de la disciplina, incluyendo la perspectiva política y social del tratamiento moral, la que nutrió los cimientos

13 En este sentido, lo positivo corresponde a la clásica dicotomía negativo/positivo y se relaciona con que históricamente las ciencias de la salud se han orientado a la enfermedad o al problema, sin considerar los factores positivos o resilientes de las personas o comunidades con quienes intervienen. No debemos confundir el término con Positivismo, ya que este término corresponde a una perspectiva epistemológica que señala que el conocimiento es siempre "positivo", es decir, que existe como entidad fuera del sujeto observador, que se acumula o suma de manera lineal y que se confirma mediante el método científico, el que sería único para todas las ciencias (3). 
de la TO, otorgándole una definición clara sobre el rol del y la terapeuta ocupacional y su trato hacia los "pacientes".

Por otro lado, el pragmatismo, epistemología presente en los inicios de la disciplina (15); (34); (35), permitió a la TO un fundamento filosófico, escasamente reconocido por la profesión, del que heredó el holismo y la crítica sobre las dicotomías para comprender el mundo. Finalmente, el movimiento de artes y oficios otorgó a la TO una perspectiva sobre el trabajo, su dignidad, valor social y el poder de la ocupación como eje de identidad en el ser humano. El tratamiento moral, enfatizando en el ser, el pragmatismo en el conocer y el movimiento de artes y oficios centrado en el hacer, forman los principios fundamentales de la primera Terapia Ocupacional.

Los dos paradigmas siguientes ya descritos, el mecanicista y de la ocupación, ejemplifican las principales epistemologías sobre la práctica de la TO, los que permiten que actualmente la TO posea diversos enfoques y áreas de acción. Antes de continuar quisiéramos hacer un alcance: siguiendo con la teoría kuhniana, el hecho de que un paradigma cese, no implica que el conocimiento posterior se elimine o ya no opere del todo, sino que se mantiene desarrollando desde sus paradigmas originales, o bien, continua hacia el paradigma siguiente. Dicho en otras palabras, el conocimiento anterior "no sigue completamente vigente", lo que en ningún término da cuenta que su contenido esté "errado", sino que implica que éste se considera de una manera distinta. Es importante recalcar este punto, debido a que el auge de una TO desde un paradigma social, o en contextos comunitarios, no implica bajo ninguna perspectiva que la TO deje de ejercerse en contextos institucionales, ni que en dichos contextos no se pueda ejercer la disciplina bajo un enfoque social. Sólo se refiere a que existe un auge en la producción de conocimiento, en el campo y ejercicio profesional, junto con líneas de investigación orientadas hacia ámbitos sociales, los que parecen tomar mayor relevancia en jornadas, congresos y seminarios.

Dicho lo anterior, continuamos con la explicación de los mapas conceptuales. Luego de los tres paradigmas mencionados aparece el cuarto: el paradigma social de la ocupación distingue el conocimiento actual de la TO y $\mathrm{CO}$, posicionando "nuevas" prácticas como válidas y novedosas. Esto no significa que antes no existieran prácticas de este tipo, sino que "cuantitativamente" eran menores, o no habían sido reconocidas por la gran comunidad de terapeutas ocupacionales $^{14}$. La TO comunitaria o la TO social, son ejemplares de este paradigma, las que se diferencian (cualitativamente) de las prácticas anteriores de la profesión y aparecen en la comunidad científica como reconocidas o como necesarias para la sociedad actual. Algo similar ocurre con "lo político" de la TO, en donde se asume que el rol y ejercicio profesional conlleva decisiones políticas inherentes a los y las terapeutas ocupacionales (38), así como en las intervenciones se desarrollan aspectos de ciudadanía centrados en los derechos humanos (40). Otro ejemplo tiene relación con la necesidad de igualdad social desde la perspectiva de género, lo que se observa en algunos artículos que intentan reivindicar el rol de las mujeres en la historia de la profesión (14); (22).

14 Un ejemplo de esto es el artículo de Oyarzun, Zolezzi, Núñez y Palacios denominado Hacia la construcción de las prácticas comunitarias de terapeutas ocupacionales en Chile, desde una mirada socio histórica, desde 1972 hasta la actualidad en la Revista Chilena de Terapia Ocupacional (36). En donde queda de manifiesto que las prácticas comunitarias y sociales, no son nuevas, sino que representan ya una larga tradición en el país. 
De esta manera, para el fortalecimiento de esta perspectiva, dentro de lo que denominamos Paradigma Social de la Ocupación, invitamos a la reflexión en torno a diferentes teorías que se condicen con el desarrollo de la TO, las que deben interpretarse desde una perspectiva compleja.

Algunas de estas teorías que consideramos fundamentales en este nuevo paradigma, son las siguientes: la teoría de la Naturaleza Ocupacional del ser humano de Ann Wilcock (29), construida a partir de reflexiones sobre la política, el ecosistema y la relación del ser humano y su medio ambiente. También, las propuestas de Michael Iwama (38), quien establece una crítica sobre los modelos de la práctica actuales de la TO, que carecen de un posicionamiento epistémico y una contextualización cultural, afectando la relación con sus sujetos de atención quienes presentan características culturales diferentes, proponiendo el Modelo Kawa. Finalmente, Frank Kronenberg, Salvador Simó Algado y Nick Pollard (40), quienes proponen un análisis reflexivo sobre sus experiencias como terapeutas ocupacionales, haciendo hincapié en la perspectiva político-social de la TO.

Por otro lado, el desarrollo de la novel Filosofía de la Ocupación Humana, la que, como hemos descrito, obedece a una reflexión de segundo orden respecto a las $\mathrm{CO}$ y TO, propone una nueva perspectiva sobre la metodología, estructura e identidad de las teorías científicas, además de permitir la introducción de estudios desde la perspectiva de género o indagando en la epistemología inicial de la profesión, la que no ha sido completamente reconocida por la TO (15); (34); (35), o que devela cómo la Ocupación, al ser un constructo, establece la interdependencia entre el sujeto y la construcción de la realidad (27), o cómo son necesarias y fundamentales las metodologías innovadoras de educación en la formación de profesionales, desde los supuestos ontológicos de la disciplina (26); (27), o cómo el estudio de la filosofía pragmatista permite un posicionamiento teórico coherente con los fundamentos que dieron origen a la TO que comprendemos hoy (15).

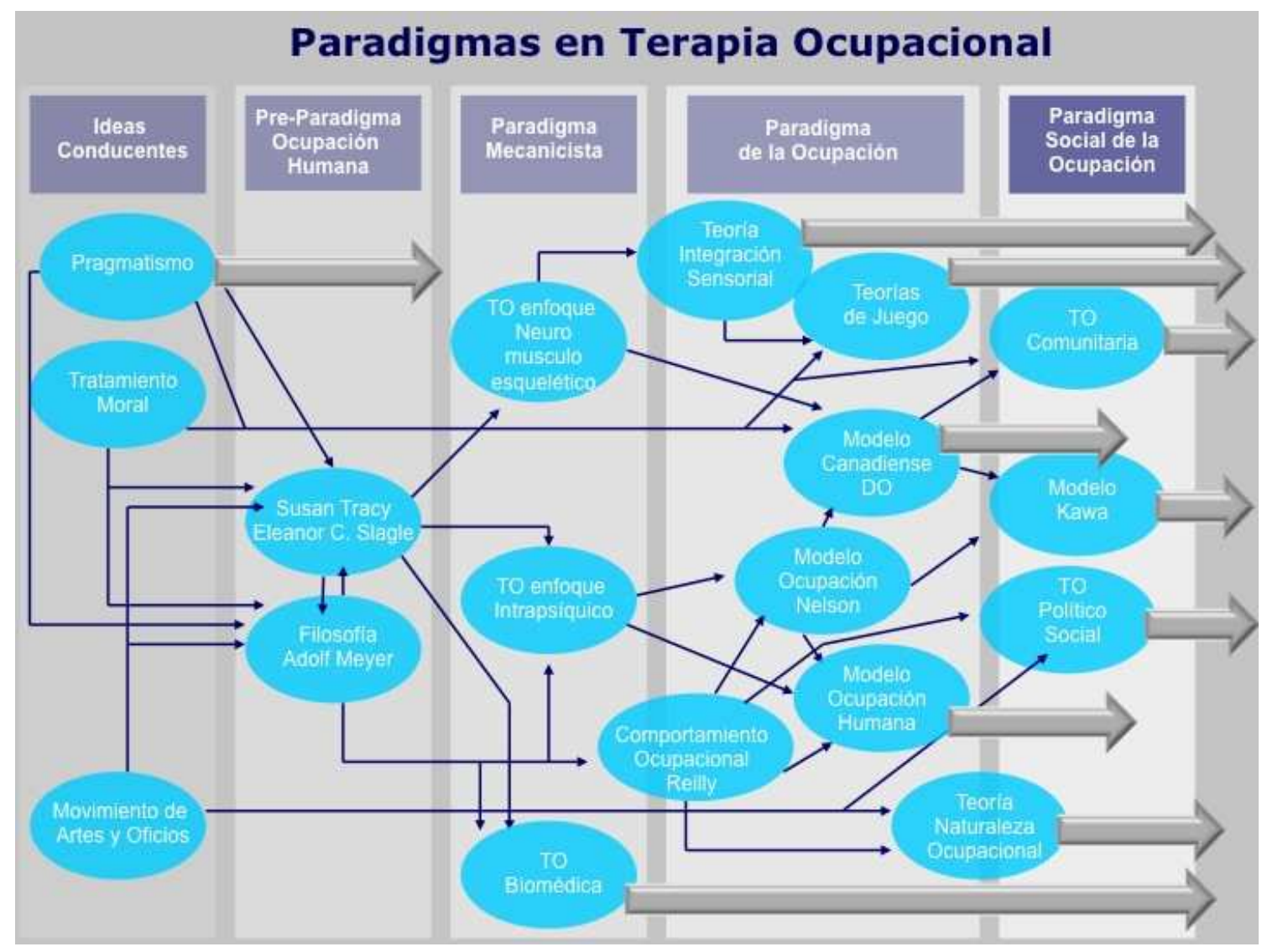


Figura 1. Representa las diferentes transiciones paradigmáticas por las que ha pasado la TO. Las teorías ejemplificadas, son sólo un referente.

Nota: Es importante señalar que el mapa conceptual no necesariamente indica todas las teorías que nutren el ejercicio profesional, son solamente ejemplares, concepto desde la teoría kuhniana que hace referencia a ejemplos o a los sellos más distintivos o populares que representan a un paradigma. Los ejemplares fueron seleccionados de acuerdo al proceso de formación y reflexión crítica de los autores y la autora, considerando solamente una pequeña muestra de todo el universo conceptual de la TO y las CO.

Finalmente, el aceptar esta invitación, no implica negar las corrientes epistemológicas más tradicionales de la TO, las cuales entregan conocimientos básicos y fundamentales para el ejercicio profesional -ciencias biomédicas-, sino por el contrario, es importante contribuir a su desarrollo y re-posicionamiento, que sea acorde a las necesidades actuales de las comunidades, de nuestras usuarias y usuarios, y también, sobre la posibilidad que la TO mantenga y supere el nivel de crecimiento y reconocimiento que está teniendo en nuestras sociedades hoy.

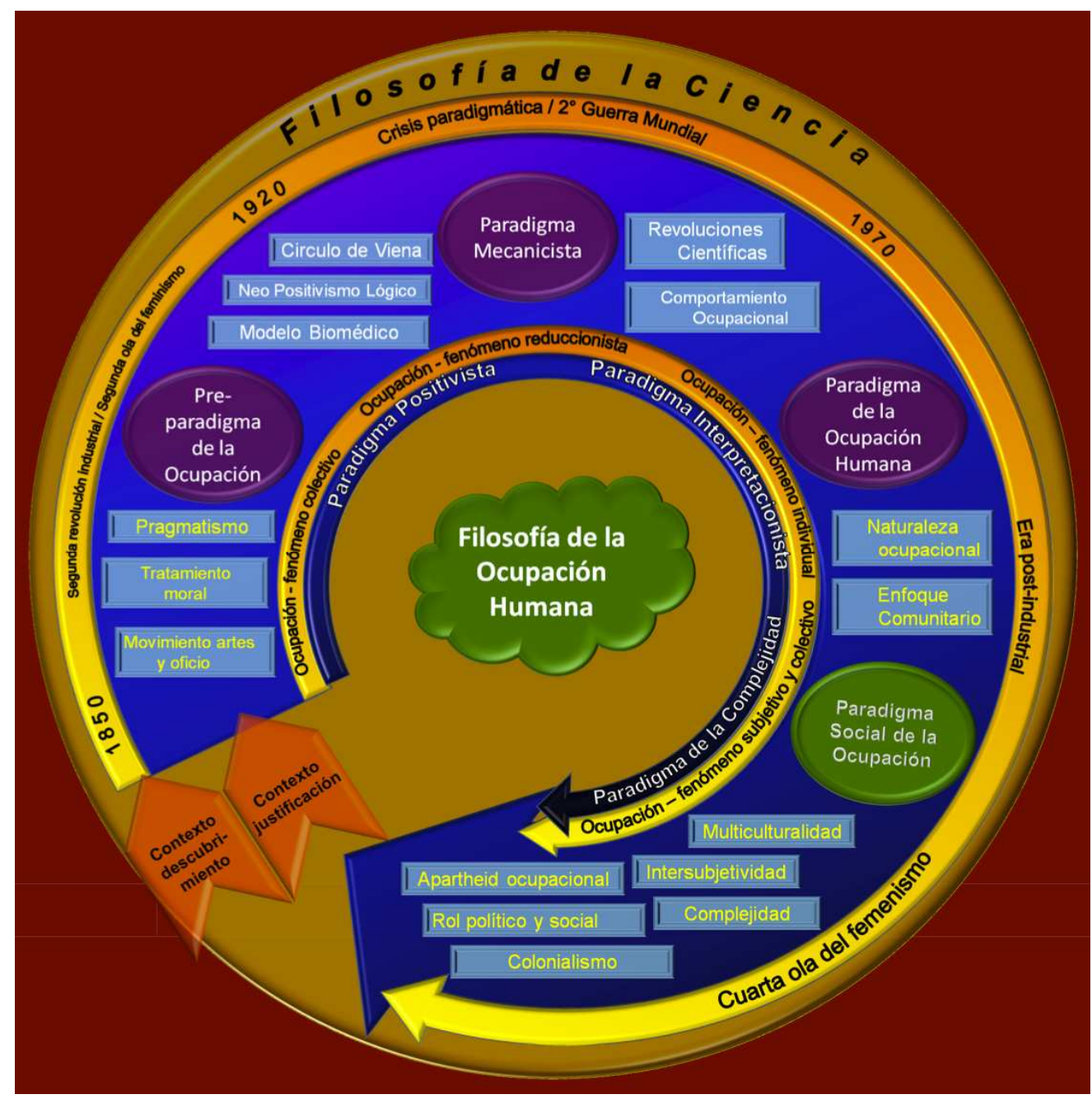

Figura 2. Representa los principales constructos teóricos que dan paso a los cambios paradigmáticos en la disciplina desde una perspectiva recursiva. 


\section{Conclusiones}

Cada paradigma se ve reflejado en la actualidad en la práctica y desarrollo teórico de la disciplina, de este modo se conceptualizan diversas terapias ocupacionales (40). El ejercicio de la profesión, en cualquiera de sus ámbitos, se vuelve tan diverso como sus sujetos de atención o como las problemáticas a abordar lo permiten, así como también la elaboración de su conocimiento científico. Queda entonces propuesto un nuevo desafío, el que invita a la reflexión, cuestionamiento y análisis de la disciplina, focalizándose siempre en cómo nuestra profesión puede mejorar el servicio que entrega a las personas, grupos y comunidades.

Además de lo anterior, continuando con las ideas de Edgar Morin en relación al logro de la coherencia y apertura epistemológica, debemos buscar meta-teorías que puedan dar cuenta de una Terapia Ocupacional que integre nuestras intervenciones y quehaceres en distintos ámbitos de acción profesional, con el objeto de dejar atrás las distinciones (falaces en opinión de los autores y autora de este artículo) entre práctica clínica, profesional, comunitaria, social, intrapenitenciaria, o cualquiera otra, pues ante el actual desarrollo del conocimiento y en palabras de Medeiros 'incluso cuando desarrolla una actividad 'simple' el terapeuta ocupacional está vehiculizando un saber y los valores ideológicos y normativos en él implícitos. Está siendo un agente social.” (32, p. 20)

Es así que, asumiendo las a veces implícitas y otras explícitas contradicciones de nuestro desempeño profesional, muchas veces poco coherentes con los principios filosóficos que dieron origen a la disciplina, creemos necesario e imprescindible que la discusión epistemológica no sólo sea una instancia a ser desarrollada en cursos de post-grado. Muy por el contrario, si deseamos "garantizar un terapeuta ocupacional pensante, crítico y dotado de herramientas para ejercer con creatividad su actividad profesional" (32, p. 16), se requiere que el pensamiento crítico y divergente sea desarrollado desde el primer día que los y las estudiantes comiencen su vida académica, por medio de la discusión epistemológica.

Dejamos establecido, entonces, un nuevo desafío el cual invita a la reflexión, cuestionamiento y análisis de la disciplina, focalizándose siempre en cómo nuestra profesión puede mejorar al servicio de las personas y comunidades. Para esto, tomamos las palabras de Morin, según el cual "la complejidad es una palabra problema y no una palabra solución" (31, p. 22), haciéndonos un llamado a la permanente revisión y desarrollo epistemológico.

\section{Agradecimientos}

A nuestras familias, ya sea de origen o la que la vida nos ha regalado, por la permanente paciencia de escuchar largas conversaciones "de segundo orden".

A los y las colegas en formación, por aceptar los desafíos compartidos desde la academia, en quienes reconocemos, desde sus trabajos, ensayos y reflexiones, nuevos aires que nos permiten seguir reflexionando y discutiendo "esto" que entendemos por realidad.

A los compañeros y compañeras de jornada, por sus constantes aportes y por aceptar el 
desafío de formar profesionales que lleguen a ser sujetos de transformación social.

A todas ellas y ellos, muchas gracias.

\section{Bibliografía y referencias}

(1) DÍEZ, J. y MOULINES, C. U. Fundamentos de Filosofía de la Ciencia. Barcelona: Ariel, 1997.

(2) ECHEVERRÍA, J. Filosofía de la Ciencia. 2da edición. Barcelona: Ariel, 1998.

(3) MARCOS, A. Ciencia y Acción. Una filosofía práctica de la ciencia. México: Fondo de Cultura Económica, 2010.

(4) LONGINO, H. The Fate of Knowledge. Princeton: Princeton University Press, 2001.

(5) CARNAP, R. Fundamentación Lógica de la Física. Buenos Aires: Sudamericana, 1969.

(6) KUHN, T. La estructura de las revoluciones científicas. México: Fondo de Cultura Económica, 1962 [1975].

(7) KOERTGE, N. A House Built on Sand: Exposing Postmodernist Myths about Science. New York: Oxford University Press, 1998.

(8) GONZÁLEZ GARCÍA, M. y PÉREZ SEDEÑO, E. Ciencia, Tecnología y Género. Revista Iberoamericana de Ciencia, Tecnología, Sociedad e Innovación. 2002, 2. Disponible en http://www.oei.es/revistactsi/numero2/varios2.htm [Consultado el 30 de octubre de 2011].

(9) BÁRCENAS, R. Contexto de descubrimiento y contexto de justificación: un problema filosófico en la investigación científica. Acta Universitaria. Universidad de Guanajuato, México, 2002. 12:2, 48-57. Disponible en: http://redalyc.uaemex.mx/redalyc/pdf/416/41600206.pdf [Consultado el 26 de octubre de 2011]

(10) MORRISON, R. Metáforas: conceptos y perspectivas para la reflexión crítica desde la Filosofía de la Ocupación Humana. Espacio T.O. Venezuela. Revista electrónica. 2011, 7. Disponible en: http://www.espaciotovenezuela.com/revista.html. [Consultado el 27 de octubre de 2011]

(11) MORRISON, R. La retórica de la ciencia. Descripciones y reflexión crítica respecto a la conformación del conocimiento. Aportes para la Terapia Ocupacional. Revista Chilena de Terapia Ocupacional, 2011, 11:1, 89-100. Disponible en: http://www.revistas.uchile.cl/index.php/RTO/article/viewFile/17085/17822. [Consultado el 27 de octubre de 2011]

(12) MORRISON, R. \& OLIVARES, D. De la filosofía, la ciencia y la filosofía...a la ocupación: propuesta epistemológica para la práctica contemporánea. Revista de Terapia Ocupacional da Universidade de São Paulo, Anais (suplemento especial). 2011, 22, out, p. 13.

(13) KIELHOFNER, G. Conceptual foundations of Occupational Therapy practice. 4th ed. Philadelphia: F. A. Davis, 2009.

(14) MORRISON, R. (Re)conociendo a las fundadoras y "madres" de la terapia ocupacional. 
Una aproximación desde los estudios feministas sobre la ciencia. TOG (A Coruña). 2011, 8, 14 [21p.]. Disponible en: http://www.revistatog.com/num14/pdfs/original4.pdf [Consultado el 24 de octubre de 2011]

(15) BREINES, E. Origins and adaptations: A philosophy of practice. Lebanon, NJ: GeriRehab, 1986.

(16) CREPEAU, E., COHN, E. \& SCHELL, B. Willard and Spackman's Occupational Therapy. 11th ed. Philadelphia: Lippincott Williams \& Wilkins, 2008.

(17) METAXAS, V. Eleanor Clarke Slagle and Susan E. Tracy: Personal and professional identity and development of occupational therapy in progressive Era America. Nursing history review. Official journal of american association for the history of nursing. 2000, 8, 39-70.

(18) QUIROGA, V. A. M. Occupational therapy: The first thirty years, 1900-1930. USA: Bethesda, MD, American Occupational Therapy Association, 1995.

(19) JAMES, W. El significado de la verdad. 4ta. ed. Buenos Aires: Aguilar Argentina, S.A. de Ediciones, 1909 [1974].

(20) FREEDMAN, E. No Turning Back: The History of Feminism and the Future of Women. USA: Ballantine Books, 2003.

(21) SLAGLE, E. C. Training aides for mental patients. Archives of Occupational Therapy. 1992, 1:1, 11-17.

(22) MORRISON, R. Eleanor Clarke Slagle. Historia y epistemología desde los estudios feministas sobre la ciencia. Cuadernos de Terapia Ocupacional da Universidade Federal de São Carlos. 2011, 19:2, Suplemento Especial. Disponible en: http://www.cbtoeclato2011.com.br/cd/resumos/TC1189-1.pdf [Consultado el 15 de octubre de 2011]

(23) DIASIO, K. Eleanor Clarke Slagle: Founder and Leader in Occupational Therapy. Occupational Therapy in Mental Health, 1985, 5:3, 101-108.

(24) MEYER, A. The Philosophy of Occupational Therapy. Archives of Occupational Therapy, 1922, 1:1, 1-10.

(25) TRACY, S. E. Studies in invalid occupation: A manual for nurses and attendants. Boston: Whitcomb \& Barrows, 1910.

(26) MORRISON, R. \& VIDAL, D. De la Filosofía a la Ciencia de la Ocupación: Propuesta epistemológica para la práctica contemporánea. En: Corregidor Sánchez, A., Romero Ayuso, D. y López Martín, O. (eds.). Práctica Clínica e Investigación en Terapia Ocupacional. España: Ed. Universidad de Castilla-La Mancha, 2011, pp. 256-7.

(27) CARRASCO, J. y OLIVARES, D. Haciendo camino al andar: construcción y comprensión de la Ocupación para la investigación y práctica de la Terapia Ocupacional. Revista Chilena de Terapia Ocupacional. 2008, 8, 5-16. Disponible en http://www.revistas.uchile.cl/index.php/RTO/article/viewArticle/55/38 [Consultado el 15 de octubre de 2011]

(28) MATTINGLY, C., \& FLEMING, M. H. Clinical reasoning: Forms of inquiry in a therapeutic practice. Philadelphia: F. A. Davis, 1994.

(29) WILCOCK, A. A. An Occupational Perspective of Health. 2nd edition. Thorofare, NJ: Slack Inc, 2006. 
(30) ZEMKE, R. \& CLARK, F. Occupational Science: The Evolving Discipline. Philadelphia: F. A. Davis Company, 1996.

(31) MORIN, E. Introducción al pensamiento complejo. Barcelona: Gedisa, 1998.

(32) DA ROCHA MEDEIROS, M. H. Terapia Ocupacional. Un enfoque epistemológico y social. Santa Fe: Universidad Nacional del Litoral, 2008.

(33) MARTÍNEZ, V. El enfoque comunitario. El desafío de incorporar a la comunidad en las intervenciones sociales. Universidad de Chile, 2006.

(34) HOOPER, B., \& WOOD, W. Pragmatism and structuralism in occupational therapy: The long conversation. American Journal of Occupational Therapy. 2002, 56, 40-50.

(35) IKIUGU M. N. \& SCHULTZ, S. An argument for pragmatism as a foundational philosophy of occupational therapy. Canadian Journal of Occupational Therapy, 2006, 73:2, 86- 97.

(36) OYARZUN, N., ZOLEZZI, R., NÚÑEZ, J. \& PALACIOS, M. Hacia la construcción de las prácticas comunitarias de terapeutas ocupacionales en Chile, desde una mirada socio histórica, desde 1972 hasta la actualidad. Revista Chilena de Terapia Ocupacional. 2009, 9, $149-165$

Disponible

en:

http://www.revistas.uchile.cl/index.php/RTO/article/viewArticle/120/103 [Consultado el 9 de Noviembre de 2011]

(37) KRONENBERG, F. \& POLLARD, N. Political dimensions of occupation and the roles of occupational therapy. American Journal of Occupational Therapy. 2006, 60:6, 617-626.

(38) IWAMA, M. The Kawa Model, Culturally Relevant Occupational Therapy. NY: Churchill Livingstone Elsevier, 2006.

(39) KRONENBERG, F., SIMÓ ALGADO, S. y POLLARD, N. Terapia Ocupacional sin Fronteras: aprendiendo del espíritu de los supervivientes. Buenos Aires: Ed. Médica Panamericana, 2006.

(40) GUAJARDO, A. y SIMÓ ALGADO, S. Una terapia ocupacional basada en los derechos humanos. TOG (A Coruña). 2010, 7, 12: [25p]. Disponible en: http://www.revistatog.com/num12/pdfs/maestros.pdf [Consultado el 20 de octubre de 2011]

(41) SOLANA, J. L. Con Edgar Morin, por un pensamiento complejo. Implicaciones interdisciplinares. Madrid: Ed. Akal, 2005.

(42) CANADIAN ASSOCIATION OF OCCUPATIONAL THERAPY. Enabling Occupation: An Occupational Therapy Perspective. Ottawa: CAOT Publications ACE, 1997.

(43) BATESON, G. Pasos hacia una ecología de la mente. Buenos Aires: Ed LOHLÉ-LUMEN, 1972 [1998]. 